\title{
Detecting Dementia-Related Wandering Locomotion of Elders by Leveraging Active Infrared Sensors
}

\author{
Qiang Lin', Weichao Zhao², Weilan Wang1 \\ ${ }^{1}$ School of Mathematics and Computer Science, Northwest Minzu University, Lanzhou, China \\ ${ }^{2}$ Tencent Technology (Beijing) Co. Ltd., Beijing, China \\ Email: qiang.lin2010@hotmail.com,1203348035@qq.com,wangweilan@xbmu.edu.cn
}

How to cite this paper: Lin, Q., Zhao, W.C. and Wang, W.L. (2018) Detecting Dementia-Related Wandering Locomotion of Elders by Leveraging Active Infrared Sensors. Journal of Computer and Communications, 6, 94-105. https://doi.org/10.4236/jcc.2018.65008

Received: April 25, 2018

Accepted: May 27, 2108

Published: May 30, 2108

Copyright $\odot 2018$ by authors and Scientific Research Publishing Inc. This work is licensed under the Creative Commons Attribution International License (CC BY 4.0).

http://creativecommons.org/licenses/by/4.0/

(c) (i) Open Access

\begin{abstract}
For elders with dementia, wandering is among the most problematic, frequent and dangerous behavior. Managing wandering behavior has become increasingly imperative due to its high prevalence, negative outcomes and burden on caregivers. We study to propose an active infrared-based method to identify wandering locomotion by monitoring rhythmical repetition of an elder's indoor motion events. Specifically, we utilize our customized active infrared sensors to collect human indoor motions that will be converted into motion events by using hardware redundancy technique. Each motion event is a directed motion obtained via introducing temporal and dimensions into the spatial motion data. Based on the most cited spatial-temporal patterns of wandering locomotion, a spatiotemporal model is then proposed to identify wandering locomotion from an ongoing sequence of motion events. Experimental evaluation on eight individuals' real-world motion datasets has shown that our proposed method is able to effectively identify wandering locomotion from repetitive events collected from active infrared sensors with a value over $98 \%$ for both accuracy and precision based on properly chosen parameters. Wandering in elders with dementia that follow specific spatiotemporal patterns can be reliably identified by analyzing repetitive motion events collected from active infrared sensors based on the well-known spatiotemporal patterns of wandering locomotion.
\end{abstract}

\section{Keywords}

Wandering Locomotion, Elders with Dementia, Active Infrared Sensors, Assisted Living

\section{Introduction}

Dementia has been identified as a progressive, disabling and chronic disease, af- 
fecting $5 \%$ of people aged above 65 and around $40 \%$ of people aged over 90 [1]. Among dementia-related behaviors, wandering is the most problematic, frequent and dangerous behaviors [2], accounting for $15 \%$ to $60 \%$ of people clinically diagnosed with dementia and other related impairments [3]. A lot of research has revealed that the frequent wanders are more vulnerable to experience adverse events, such as falling, elopement, boundary transgression and getting lost [4] [5]. Wandering manifests itself as aimless movement, repetitive locomotion with no identifiable goal and disorientation to time and place [6] [7]. Thus, managing wandering behavior of people with dementia has become increasingly imperative due to its high prevalence, negative outcomes and burden on caregivers [8].

Traditional solutions to prevent elders with dementia from wandering mainly include imposing physical restraints and/or using medicine. However, the physical or psychological problems caused by physical restraint and the side-effects of neuroleptic drugs make the traditional methods infeasible or ineffective to protect wanderers especially for those individuals who are prone to falling or unsafe wandering [9].

Preventive measures able to maximize autonomy while minimizing risk are recommended to monitor wandering-prone elders in home, community and care facility environments [10]. The main purpose of preventive measures is to manage rather than prevent wandering by highlighting a shift from prevention towards assisting safe walking [11].

A large number of sensors-based solutions for managing wandering behavior have been proposed by using different techniques [12], which can be categorized into three main categories: the event monitoring based methods, the trajectory tracking based methods and the localization combined with Geofencing techniques. In this work, we propose to develop an infrared-based method to identify the wandering-related rhythmical repetition of motion events in indoor environment for elders with dementia living alone. The proposed method differs from the existing work in three aspects.

- First, active infrared (AIR) sensors are used to sense human indoor motions when an individual walks into/out rooms or move across certain crucial area. Compared to those existing systems developed by using switch sensors, UWB sensors, step-count sensors, passive-RFID, pressure sensors [13] [14] [15] [16] and GPS [17] [18] [19] for outdoor setting, the AIR sensors based system is more affordable, scalable and easy to deploy.

- Second, all the spatial and temporal dimensions of human motions are integrated to build a spatiotemporal wandering model based on the spatiotemporal patterns of wandering [20]. The built spatiotemporal model can identify wandering-related anomalous motions while reducing the false positives.

- Last, more than one thousand sequences of motions were used to evaluate the proposed method. Experimental results have shown that the proposed method obtains good performance for detecting wandering locomotion with a value over $98 \%$ for both accuracy and precision, respectively. 
The rest of this paper is organized as follows. In Section 2, we present our proposed method for detecting indoor wandering behavior using AIR based system. In Section 3, we provide the experimental evaluation results conducted on real-world datasets from eight individuals. And in Section 4, we conclude this work.

\section{Methodology}

To keep the completeness, in this Section, we first provide the most cited definition and spatiotemporal patterns for wandering behavior.

Although different attempts have been done for defining wandering behavior, there is no a widely accepted definition so far because wandering behavior is very complex and occurs for a number of reasons. We herein present the latest one proposed by Algase et al. [21] which defines wandering as follows: a syndrome of dementia-related locomotion behavior having a frequent, repetitive, temporally-disordered, and/or spatially-disoriented nature that is manifested in lapping, random, and/or pacing patterns, some of which are associated with eloping, eloping attempts, or getting lost unless accompanied.

We can see from the above definition, wandering locomotion is characterized by its anomalies on walking frequency, repetition and spatiotemporal disorientation. Especially, the spatial aspect of wandering behavior can be divided into several different patterns (lapping, pacing and random patterns) [20]:

- Direct pattern: walking directly from one place to another without any detour;

- Pacing pattern: back and forth motion between any two points, i.e., physical locations;

- Lapping pattern: circuitous motion revisiting some points sequentially along a path;

- Random pattern: haphazard motion without repeating points in traveling sequence.

Among the above patterns, the direct one is regarded as normal and the remaining patterns link to wandering behavior. Figure 1 illustrates the spatial patterns of wandering.

Algase [22] represented wandering movement as spatiotemporal locomotion by introducing temporal factor into the above spatial patters. The term locomotion refers to rhythmical motions consisting of two phases: walking and non-walking (as shown in Figure 2). During the walking phase, the person would wander following one or more of the pacing, lapping and random patterns. After every walking phase, there will be a non-walking duration of different time interval.

\subsection{Acquiring Human Motion Events}

Detecting wandering locomotion by using active infrared sensors is consisted into two main steps. The first step is responsible for acquiring human indoor motions with active infrared sensors. The second step is in charge of detecting 


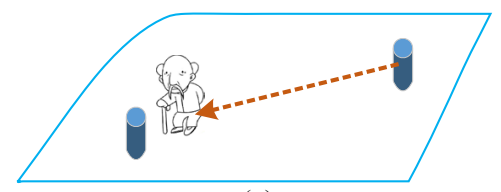

(a)

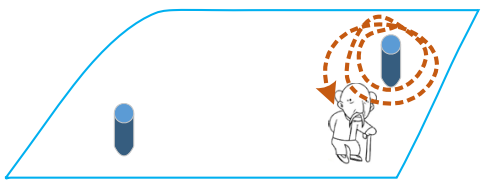

(c)

- Locations such as rooms or crucial areas

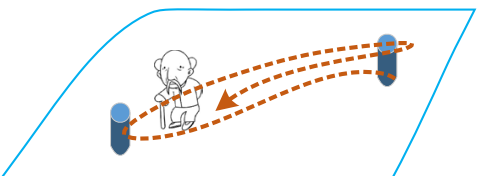

(b)

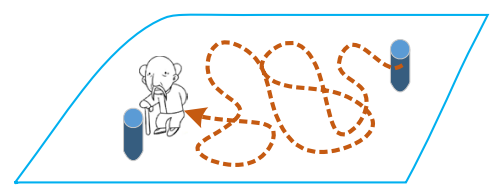

(d)

Persons such as elders

Figure 1. The spatial patterns of wandering movement behavior. (a) Direct pattern; (b) Pacing pattern; (c) Lapping pattern; (d) Random pattern.

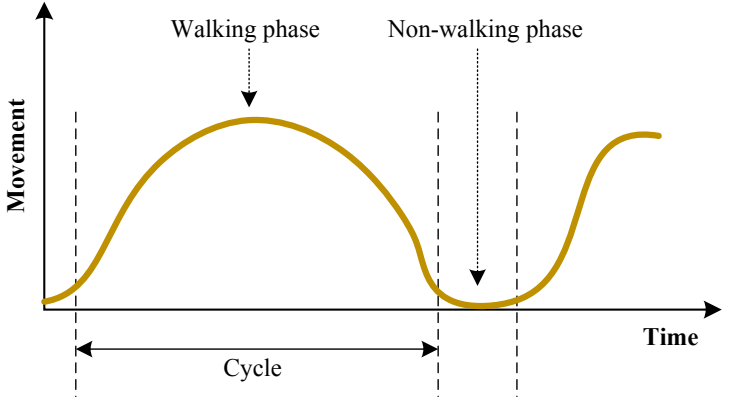

Figure 2. Wandering locomotion consisting of walking and non-walking phases.

wandering locomotion through analyzing rhythmical repetition of events from the monitored motions in the first step.

The proposed method uses our customized AIR sensors to monitor human indoor motions. As depicted in Figure 3, an AIR sensor consists of two-piece elements with one containing an infrared transmitter and another containing an infrared receiver. To obtain a reliable detection of human indoor motions, the transmitter and receiver of an AIR sensor are required to be accurately aligned.

In the customized AIR-based sensor system, the infrared transmitter sends a beam of infrared light (essentially invisible due to its longer wavelengths than those of visible light) which will be received by the infrared receiver. Generally, a continuous signal can be received by the receiver if there is no obstruction (both the static and mobile) between the transmitter and receiver. On the contrary, when a mobile object moves across the scan area of a transmitter, the beam of infrared light will be interrupted (as depicted in Figure 3). That is exactly what we are applying in this work to detect human indoor motion by using AIR based sensors. Specifically, let $d$ denote the duration of a beam of infrared light that was interrupted by some motion, if $d_{L} \leq d \leq d_{U}$, this motion will be recognized as a motion caused by an elder entering or exiting a door or a functional area. Formally, a detected human motion can be represented by Equation (1):

$$
\text { motion }=(s, t)
$$




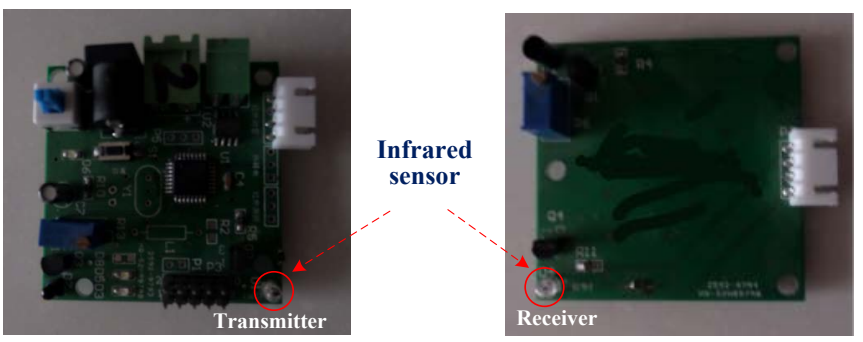

Figure 3. The customized active infrared sensor for monitoring human indoor motions.

where $s$ denotes the sensor that an elder traverses at time $t$. An elder's continuous motions will produce a sequence of motions, i.e., $\left\{\right.$ motion $_{1}$, motion $_{2}, \ldots$, motion $\left._{i}, \ldots\right\}$, with motion $_{i}$ denoting the $i$-th motion. Otherwise, further processes are needed as follows.

- If $d \leq d_{L}$, a motion is detected which is often caused by infrared-sensitive burst interferences (e.g., transient bright light). The motion, in this case, often has obviously shorter duration than the one of human motion.

- If $d \geq d_{U}$, a motion is detected which is often caused by relatively-persistent interferences (e.g., dense smoke). The motion in this case often has obviously larger duration than the one of human motion.

In this paper, the values for $d_{L}$ and $d_{U}$ are experimentally determined based on the average human walking speed of 5.0 kilometers per hour by fully taking elders' health conditions into account. With a detected motion by leveraging AIR sensor, the following task is to determine the motion-related event. In this work, a motion-related event is semantically defined as a directed motion, which corresponds to entering/exiting a room or a crucial area by an elder. Specifically, we consider only two directions for the directed motions: entering and exiting. Thus, an event (i.e., directed motion) can be represented as follows.

$$
\text { event }=(\text { motion }, d r t)
$$

where $d r t=($ in, out $)$ refers to the set of the directions, and in and out denotes the entering and exiting, respectively. We propose a hardware redundancy technique to acquire an event from one detected motion. The hardware redundancy technique is utilized to integrate two pairs of transmitters and receivers in an AIR sensor. For an AIR sensor consisting of T1-R1 and T2-R2, we need to ensure that the scan zones of T1 and T2 are not overlap with each other (see Figure 4(a)). Now, if a motion that first traverses the scan zone of $\mathrm{T} 1$ and then the one of $\mathrm{T} 2$ is defined as an entering event, the opposite motion from $\mathrm{T} 2$ to $\mathrm{T} 1 \mathrm{can}$ be therefore regarded as an existing event as shown in Figure 4(b).

\subsection{Detecting Wandering Locomotion}

With the spatial wandering patterns as mentioned previously, a wandering locomotion can be regarded as a sequence of events that follow one or more spatial patterns. To build a spatio-temporal model for detecting wandering behavior, we need to examine the temporal factors of such a behavior. 


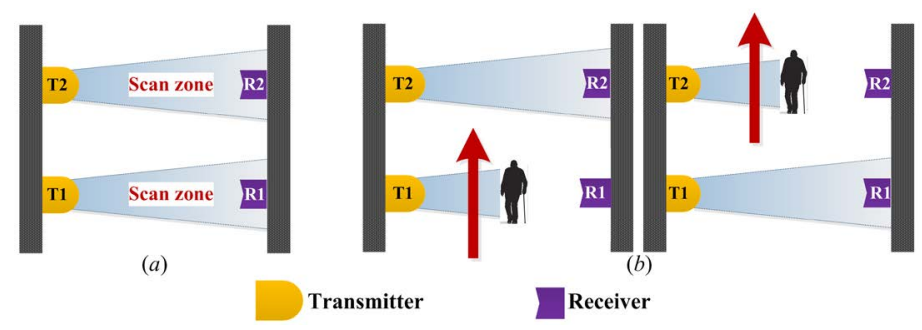

Figure 4. The hardware redundancy technique for acquiring human indoor motion event. (a) The redundant transmitters (T1 and T2) and receivers (R1 and R2) with no overlap of scan zones of T1 and T2; (b) An example of detecting a motion event.

Given an event sequence of $\left\{\ldots\right.$, motion $_{i-2}$, motion $\left._{i-1}\right\}$ that follows one of the pacing, lapping and random spatial patterns. For a new event motion $_{i}$ if the time difference $g_{i}=t_{i}-t_{i-1}$ of these two events motion $_{i-1}$ and motion $_{i}$ is larger than a given threshold $g_{T}$, a finished event sequence $\left\{\ldots\right.$, motion $_{i-2}$, motion $\left._{i-1}\right\}$ has been detected. Otherwise, we obtain an ongoing event sequence $\left\{\ldots\right.$, motion $_{i-2}$, mo- $^{-}$ $\operatorname{tion}_{i-1}$, motion $_{i}$. It is worth noting that the threshold $g_{T}$ is experimentally determined. For any one shortest event sequence, such as $\left\{\right.$ motion $\left._{i-1}, \operatorname{motion}_{i}\right\}$, we can calculate a score for it as $\operatorname{score}_{i}=1 / g_{i}$ Obviously, the lower the time difference $t_{i}-t_{i-1}$ is, the larger the score score $_{i}$ achieves. The reason is that wandering locomotion is always frequent, thus the small value of $t_{i}-t_{i-1}$. Therefore, for a sequence consisting of $n$ events, the total score can be calculated according to Equation (3).

$$
\text { score }=\sum_{i=1}^{n} \text { score }_{i}
$$

Now, we obtain a spatiotemporal wandering model as follows.

$$
\text { Wand }=(S, T) \mid \begin{aligned}
& S=\{\text { Lapping, Pacing, Random }\} \\
& T=\left\{g_{T}, \text { score }\right\}
\end{aligned}
$$

where $S$ is the spatial set consisting of all spatial patterns that a wandering locomotion may follow, and $T$ is the temporal set containing threshold and score for an event sequence. Algorithm 1 provides the algorithm for detecting wandering locomotion based on repetitive events collected from the AIR sensors in indoor environment.

In Algorithm 1, the Spatial_Wand procedure is used to identify the spatial pattern of an event sequence, which will be presented in Algorithm 2. For a given event sequence consisting of $n$ events, if Algorithm 1 outputs $n-1$ " $L$ " (" $P$ "), a wandering locomotion that follows the lapping (pacing) pattern has been detected. Otherwise, a wandering locomotion that follows random pattern has been detected.

\section{Experimental Evaluations}

\subsection{Experimental Setup}

In the evaluation, we recruited eight volunteers to collect motion data by using 
our customized AIR sensors. Overall, there are totally eight datasets collected from eight volunteers. In each dataset, there are the number of the total sequences, the number of the sequences following the lapping, pacing and random patterns, and the wandering ratio of the wandering sequences in the total sequences (as shown in Table 1).

The direct sequences in Table 1 are those normal motions without wandering. Because of the realistic difficulty of collecting motion data from the elders with dementia, the recruited volunteers are those healthy individuals. Before collecting data, all the eight volunteers are well trained beforehand with the spatiotemporal characteristics of wandering locomotion. Then, every volunteer is asked to wander following different patterns of wandering (i.e., direct, lapping, pacing and random), which leads to a motion dataset consisting of motions with different spatiotemporal patterns.

Algorithm 1. The event-based wandering detecting algorithm.

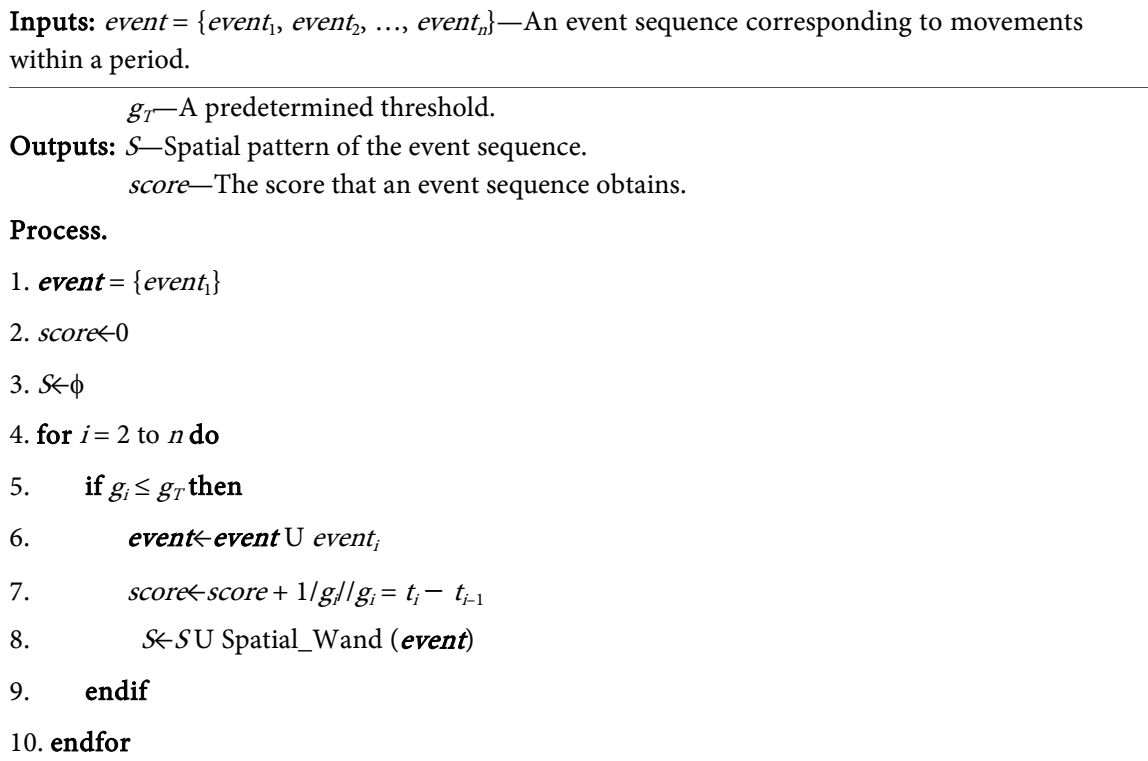

Algorithm 2. The Spatial_Wand procedure.

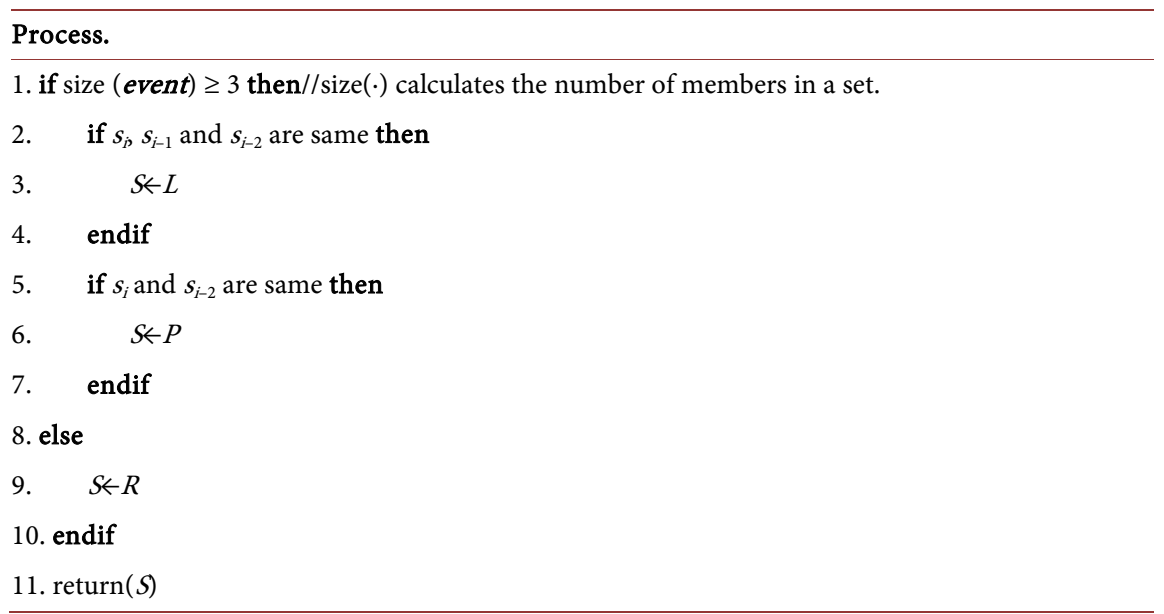


Table 1. An overview of the collected motion datasets.

\begin{tabular}{ccccccccc}
\hline \multirow{2}{*}{$\begin{array}{c}\text { Motion } \\
\text { sequences }\end{array}$} & \multicolumn{7}{c}{ Datasets } \\
\cline { 2 - 9 } & D-1 & D-2 & D-3 & D-4 & D-5 & D-6 & D-7 & D-8 \\
\hline The total number & 143 & 152 & 146 & 140 & 153 & 146 & 144 & 145 \\
Direct & 64 & 48 & 74 & 94 & 57 & 49 & 58 & 47 \\
Random & 42 & 44 & 48 & 34 & 63 & 69 & 57 & 57 \\
Pacing & 25 & 36 & 15 & 8 & 15 & 16 & 20 & 26 \\
Lapping & 12 & 24 & 9 & 4 & 18 & 12 & 9 & 15 \\
Wandering Ratio & $55.24 \%$ & $68.42 \%$ & $49.32 \%$ & $32.86 \%$ & $62.75 \%$ & $66.44 \%$ & $59.72 \%$ & $67.59 \%$ \\
\hline
\end{tabular}

Figure 5 demonstrates the layout of our experimental system in an apartment, consisting of one sitting room, one kitchen, one study room, one bathroom and two bedrooms. Each room was deployed with an AIR sensor on the door at a height of 1.5 meter as indicated by red solid rectangles (as shown on the lower branch in Figure 5). Furthermore, in order to obtain fine-grained motion sequences, we deploy some crucial areas with AIR sensors as indicated by green solid circles (as shown on the upper branch in Figure 5). Finally, total eleven groups of AIR sensors were deployed in the system.

Furthermore, in order to quantitatively evaluate the proposed method, we asked three geriatric domain experts to manually label all the sequences in each dataset. If the majority of the experts (i.e., at least two of them) think that a motion sequence is an outlying motion event (i.e., probably a wandering locomotion), it is labelled as an outlying one. The volunteers comprise of one male geriatrician (ages 55) and two physicians (ages 43 and 27 respectively). Three experts independently labelled each sequence after being trained to identify three different patterns of wandering locomotion. These manually labelled outlying events serve as the ground truth in the experiments.

The evaluation metric we use is accuracy and precision. In practice, a detected sequence falls into one of the four categories:

- True Positive (TP), which correctly identifies an outlying sequence as an outlier;

- False Positive $(F P)$, which incorrectly identifies a normal sequence as an outlier;

- False Negative $(F N)$, which incorrectly identifies an outlying sequence as normal; and

- True Negative $(T N)$, which correctly identifies a normal sequence as normal. Accordingly, we define the accuracy acc and precision pre as follows:

$$
a c c=\frac{T P+T N}{T P+F P+T N+F N}, \text { and } p r e=\frac{T P}{T P+F P} .
$$

The experiments are run in Matlab on an Intel Core i7-6500U PC with 8 GB RAM running Windows 10 . 


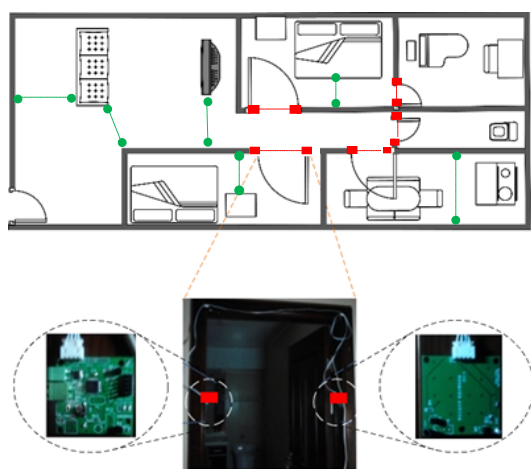

Figure 5. The deployment of our AIR sensor based system.

\subsection{Experimental Results}

We quantitatively evaluate the proposed method by calculating its accuracy and precision values. The values for parameters $d_{L}$ and $d_{U}$ are set as 0.5 second and 1.5 seconds respectively. In addition, the value of threshold $g_{T}$ is set as 2 minutes. It means that for an already arrived event sequence $\left\{\ldots\right.$, motion $_{i-2}$, motion $\left._{i-1}\right\}$ and a new motion $_{i}$ if the duration of motion $_{i-1}$ and motion $_{i}$ is larger than $2 \mathrm{mi}$ nutes, we have a finished event sequence $\left\{\right.$ motion $_{1} \ldots$, motion $\left._{i-2}, \operatorname{motion}_{i-1}\right\}$ and a new sequence has already started at motion $_{i}$.

Table 2 provides the experimental results about accuracy acc and precision pre for all eight datasets, from which we can see that almost all the datasets obtained a value of more than $98 \%$ for both accuracy and precision.

In order to differentiate all the wandering events with different spatiotemporal patterns, Figure 6 demonstrates a comparation between the ground truth and the detected ones for all datasets. Intuitively, our proposed method obtained a comparable performance. Particularly, the events following the lapping pattern have almost exactly same curves as shown in Figure 6(d).

\subsection{Discussion}

Experimental evaluation conducted on eight individuals' real-world motion datasets has demonstrated that our proposed method achieved a value of over $98 \%$ for both accuracy and precision on detecting wandering locomotion based on the properly chosen parameters. However, we need to pay attentions to the following two situations when apply our proposed method for detecting dementia-related wandering of elders.

- First, an event sequence following lapping pattern is uniquely associated with a certain sensor, and an event sequence following pacing pattern may contain one or more pacing events. As a result, the events following lapping patterns can always be detected by using our AIR sensors.

- Second, in practice, there is often relatively small difference between the direct and random patterns because an event sequence corresponding to doing housework may move across several areas or doors, leading to misdetection between the direct and random motions. 
Table 2. The average accuracy and precision for all eight datasets.

\begin{tabular}{ccccccccccc}
\hline & \multicolumn{2}{c}{ Direct } & \multicolumn{2}{c}{ Random } & \multicolumn{2}{c}{ Pacing } & \multicolumn{2}{c}{ Lapping } & \multirow{2}{*}{ Accuracy } & Precision \\
\cline { 1 - 5 } & Gt & Dt & Gt & Dt & Gt & Dt & Gt & Dt & & \\
\hline D-1 & 64 & 63 & 42 & 44 & 25 & 24 & 12 & 12 & 0.9931 & 0.9877 \\
D-2 & 48 & 46 & 44 & 45 & 36 & 37 & 24 & 24 & 0.9870 & 0.9815 \\
D-3 & 74 & 72 & 48 & 47 & 15 & 19 & 9 & 8 & 0.9865 & 0.9737 \\
D-4 & 94 & 94 & 34 & 35 & 8 & 7 & 4 & 4 & 1.0000 & 1.0000 \\
D-5 & 57 & 55 & 63 & 67 & 15 & 14 & 18 & 17 & 0.9871 & 0.9800 \\
D-6 & 49 & 47 & 69 & 70 & 16 & 17 & 12 & 12 & 0.9865 & 0.9802 \\
D-7 & 58 & 57 & 57 & 60 & 20 & 18 & 9 & 9 & 0.9931 & 0.9886 \\
D-8 & 47 & 46 & 57 & 57 & 26 & 27 & 15 & 15 & 0.9932 & 0.9900 \\
\hline
\end{tabular}

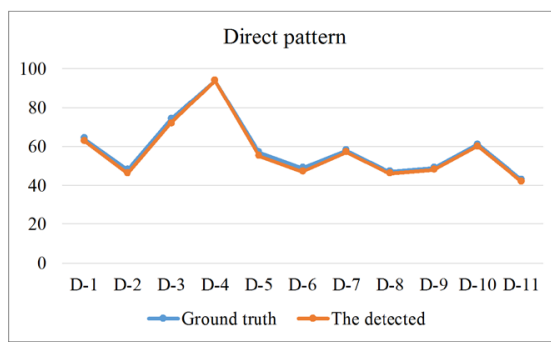

(a)

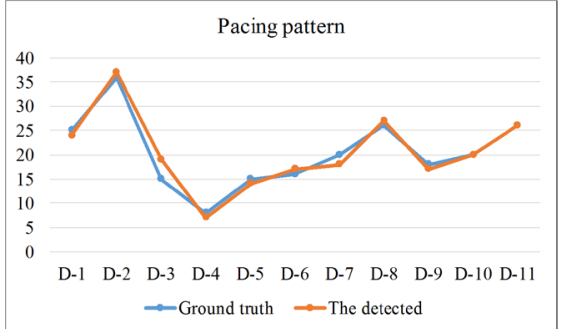

(c)

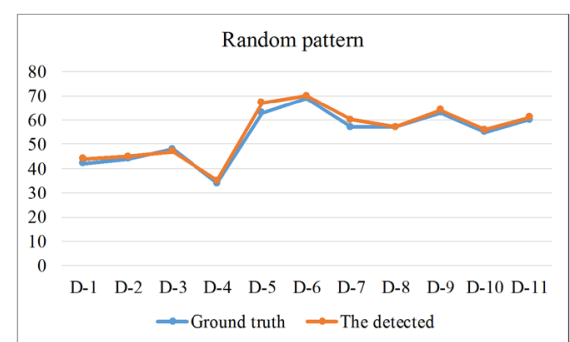

(b)

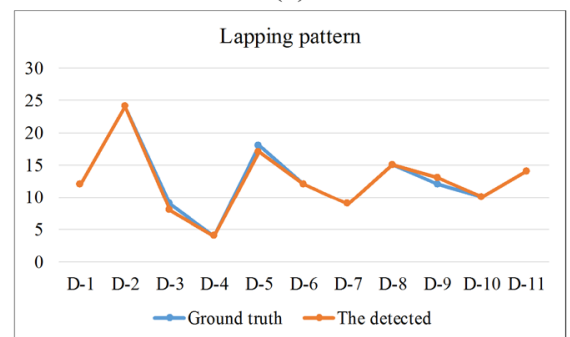

(d)

Figure 6. The detected vs. the ground truth for all datasets. (a) The direct pattern; (b) The random pattern; (c) The pacing pattern; (d) The lapping pattern.

\section{Conclusions}

In this paper, we have proposed an AIR based method for detecting elders' wandering behavior, where a group of customized AIR sensors were used to monitor elders' indoor motions. With the monitored motions, a hardware redundancy technique has been proposed to convert each motion into a motion event. Then a spatiotemporal wandering model has been proposed to identify wandering locomotion from an ongoing sequence of motion events based on the spatial-temporal patterns of wandering behavior.

Using more than one thousand motion sequences collected from eight volunteers, our proposed method has been evaluated to examine its effectiveness on detecting wandering-related anomalous movement by analyzing repetitive motion events. The experimental results show that our proposed method is applica- 
ble to the event-based detecting of wandering locomotion by using AIR sensors. A value of over $98 \%$ for both accuracy and precision has been achieved based on properly chosen parameters.

In summary, wandering in elders with dementia that follows specific spatiotemporal patterns can be reliably identified by analyzing repetitive motion events that are collected from AIR sensors based on the well-known spatiotemporal patterns of wandering locomotion.

\section{Acknowledgements}

The authors would like to thank all the anonymous reviewers and the editor-in-chief. This work is partially supported by the National Natural Science Foundation of China (No. 61562075), the Natural Science Foundation of Gansu Province (No. 1506RJZA269), the Fundamental Research Funds for the Gansu Universities (No. 2015B-002), the Fundamental Research Funds for the Central Universities (Nos. 31920150081, 31920180114) and the Gansu Provincial First-Class Discipline Program of Northwest Minzu University.

\section{References}

[1] Hendrie, H.C. (1998) Epidemiology of Dementia and Alzheimer's Disease. American Journal of Geriatric Psychiatry, 6, S3-S18. https://doi.org/10.1097/00019442-199821001-00002

[2] Teri, L., Larson, E.B. and Reifler, B.V. (1988) Behavioral Disturbance in Dementia of the Alzheimer's Type. Journal of the American Geriatrics Society, 36, 1-6. https://doi.org/10.1111/j.1532-5415.1988.tb03426.x

[3] Chan, D.C., Kasper, J.D., Black, B.S. and Rabins, P.V. (2003) Prevalence and Correlates of Behavioral and Psychiatric Symptoms in Community-Dwelling Elders with Dementia or Mild Cognitive Impairment: The Memory and Medical Care Study. International Journal of Geriatric Psychiatry, 18, 174-182. https://doi.org/10.1002/gps.781

[4] Siders, C., Nelson, A., Brown, L.M., Joseph, I., Algase, D., Beattie, E., et al. (2004) Evidence for Implementing Nonpharmacological Interventions for Wandering. Rehabilitation Nursing, 29, 195-206.

[5] Vassallo, M., Poynter, L., Sharma, J.C., Kwan, J. and Allen, S.C. (2008) Fall Risk-Assessment Tools Compared with Clinical Judgment: An Evaluation in a Rehabilitation Ward. Age \& Ageing, 37, 277-281. https://doi.org/10.1093/ageing/afn062

[6] Vuong, N.K., Chan, S. and Lau, C.T. (2015) mHealth Sensors, Techniques, and Applications for Managing Wandering Behavior of People with Dementia: A Review. Mobile Health. In: Adibi, S., Ed., Mobile Health, Springer, Cham, 11-42.

[7] Dphil, V.L.P. and Dphil, S.D. (2003) The Incremental Effect of Dementia-Related Problem Behaviors on the Time to Nursing Home Placement in Poor, Frail, Demented Older People. Journal of the American Geriatrics Society, 51, 188-193. https://doi.org/10.1046/j.1532-5415.2003.51057.x

[8] Sansrimahachai, W. (2016) Stream-Based Wandering Monitoring System for Elderly People with Dementia. International Symposium on Communications and Information Technologies, Nara, 7-9 October 2015, 1-4.

[9] Moore, D.H. and Algase, D.C.G. (2009) A Framework for Managing Wandering 
and Preventing Elopement. American Journal of Alzheimer's Disease \& Other Dementias $^{\circledR}, 24,208-219$. https://doi.org/10.1177/1533317509332625

[10] Laney, L. and Davey, D.B. (2009) Dementia and Wandering Behavior: Concern for the Lost Elder. Issues in Mental Health Nursing, 25, 553-555.

https://doi.org/10.1080/01612840490443482

[11] Cohen-Mansfield, J. and Werner, P. (1998) The Effects of An Enhanced Environment on Nursing Home Residents Who Pace. The Gerontologist, 38, 199-208. https://doi.org/10.1093/geront/38.2.199

[12] Lin, Q., Zhang, D., Chen, L., Ni, H. and Zhou, X. (2014) Managing Elders' Wandering Behavior Using Sensors-Based Solutions: A Survey. International Journal of Gerontology, 8, 49-55. https://doi.org/10.1016/j.ijge.2013.08.007

[13] Doughty, K., Williams, G., King, P.J. and Woods, R. (1998) DIANA-A Telecare System for Supporting Dementia Sufferers in the Community. International Conference on Engineering in Medicine and Biology Society, Hong Kong, 1-1 November 1998, 1980-1983.

[14] Ota, K., Ota, Y., Otsu, M. and Kajiwara, A. (2011) Elderly-Care Motion Sensor Using UWB-IR. Sensors Applications Symposium, San Antonio, 22-24 February 2011, 159-162. https://doi.org/10.1109/SAS.2011.5739786

[15] Masuda, Y., Yoshimura, T., Nakajima, K. and Nambu, M. (2002) Unconstrained Monitoring of Prevention of Wandering the Elderly. Engineering in Medicine and Biology, 2002. Conference and the Fall Meeting of the Biomedical Engineering Society EMBS/ BMES Conference, Houston, 23-26 October 2002, 1906-1907. https://doi.org/10.1109/IEMBS.2002.1053086

[16] Jit, B., Zhang, D., Qiao, G., Foo, V., Qiu, Q. and Yap, P. (2006) A System for Activity Monitoring and Patient Tracking in a Smart Hospital. International Conference on Smart Homes and Health Telematics, Belfast, 25-25 May 2006, 196-203.

[17] Kumar, A., Ma, M., Lau, C.T. and Chan, S. (2017) A Framework of Real-time Wandering Management for Person with Dementia. International Conference on Computer Modeling and Simulation, Canberra, 20-23 January 2017, 146-150.

[18] Helmy, J. and Helmy, A. (2017) Demo Abstract: Alzimio: A Mobile App with Geofencing, Activity-Recognition and Safety Features for Dementia Patients. 2017 IEEE Conference on Computer Communications Workshops, Atlanta, 1-4 May 2017, 994-995. https://doi.org/10.1109/INFCOMW.2017.8116527

[19] Helmy, J. and Helmy, A. (2016) The Alzimio App for Dementia, Autism \& Alzheimer's: Using Novel Activity Recognition Algorithms and Geofencing. IEEE International Conference on Smart Computing, St. Louis, 18-20 May 2016, 1-6.

[20] Martino-Saltzman, D., Blasch, B.B., Morris, R.D. and Mcneal, L.W. (1991) Travel Behavior of Nursing Home Residents Perceived as Wanderers and Nonwanderers. The Gerontologist, 31, 666-672. https://doi.org/10.1093/geront/31.5.666

[21] Algase, D.L., Moore, D.H., Vandeweerd, C. and Gavin-Dreschnack, D.J. (2007) Mapping the Maze of Terms and Definitions in Dementia-Related Wandering. $\mathrm{Ag}$ ing \& Mental Health, 11, 686-698. https://doi.org/10.1080/13607860701366434

[22] Algase, D.L. (1999) Wandering. A Dementia-Compromised Behavior. Journal of Gerontological Nursing, 25, 10-16. https://doi.org/10.3928/0098-9134-19990901-06 